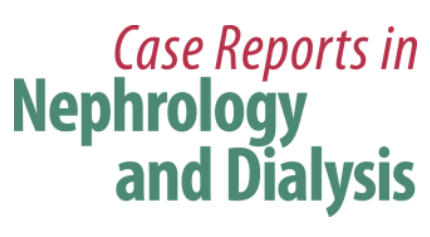

\title{
Radical Improvement of Signs and Symptoms in Systemic Lupus Erythematosus when Treated with Hemodiafiltration with Endogenous Reinfusion Dialysis
}

\author{
Francesco Giuseppe Solano $^{a} \quad$ Elisa Bellei $^{\mathrm{b}, \mathrm{c}} \quad$ Aurora Cuoghi $\mathrm{i}^{\mathrm{b}, \mathrm{c}}$ \\ Marialuisa Caiazzo ${ }^{d}$ Francesco Bruni $^{\mathrm{a}}$ \\ ${ }^{a}$ Nephrology and Dialysis, Civil Hospital Madonna del Soccorso, San Benedetto del \\ Tronto, ${ }^{b}$ Department of Diagnostic, Clinical and Public Health Medicine, University of \\ Modena and Reggio Emilia, Modena, 'Science and Technology Park for Medicine-TMP, \\ Democenter Foundation, and ${ }^{d}$ Scientific Affairs, Bellco S.r.I., Mirandola, Italy
}

\section{Key Words}

Lupus nephritis · Antiphospholipid syndrome $\cdot$ Hemodiafiltration with endogenous reinfusion · High-performance liquid chromatography coupled with quadrupole time-of-flight mass spectrometer

\begin{abstract}
Lupus nephritis is one of the most serious complications of systemic lupus erythematosus (SLE). In the kidney, immune complexes and autoantibodies activate mesangial cells that secrete cytokines that can further amplify inflammatory processes. We present the case of a 42-year-old woman with lupus nephritis accompanied by periods of exacerbation of SLE, with necrotic-like skin lesions, psoriatic arthritis without skin psoriasis, purpura of the lower limb, petechial rash, joint pain, fever, eyelid edema with bilateral conjunctival hyperemia and itching. The patient underwent a dialytic treatment of hemodiafiltration with endogenous reinfusion. The technique uses the super-high-flux membrane Synclear 02 (SUPRA treatment) coupled with an adsorbent cartridge that has affinity for many toxins and mediators. Fever and joint pain were immediately reduced after treatment and, subsequently, there was a notable reduction of the skin damage. Prednisone and immunosuppressive drugs were gradually reduced until complete suspension. High-performance liquid chromatography coupled with quadrupole time-of-flight mass spectrometer was performed for identification of proteins
\end{abstract}

KARGER 125/s $\quad \begin{aligned} & \text { Francesco Bruni } \\ & \text { Nephrology and Dialysis } \\ & \text { Civil Hospital Madonna del Soccorso, Via Silvio Pellico } 7 \\ & \text { IT-63074 San Benedetto del Tronto, Ascoli Piceno (Italy) } \\ & \text { E-Mail Francesco.Bruni@sanita.marche.it }\end{aligned}$




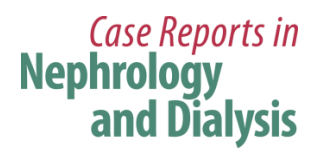

Case Rep Nephrol Dial 2015;5:106-112

DOI: $10.1159 / 000381395$

(c) 2015 S. Karger AG, Basel

www.karger.com/cnd

Solano et al.: Radical Improvement of Signs and Symptoms in SLE when Treated with Hemodiafiltration with Endogenous Reinfusion Dialysis

captured by a resin bed during a dialysis session of the patient. This technique identified several biomarkers of kidney injuries, uremic toxins, fragments of immunoglobulins, antigens involved in antiphospholipid syndrome and a new marker ( $\alpha$-defensin) that correlated significantly with disease activity. The removal of these different proteins could possibly provide an explanation of the improvement in the patient's symptoms and the normalization of her SLE. SUPRA coupled with an adsorption may be a promising new technique for the treatment of lupus nephritis.

(C) 2015 S. Karger AG, Basel

\section{Introduction}

Lupus nephritis (LN) is one of the most severe manifestations of systemic lupus erythematosus (SLE). The clinical course ranges from asymptomatic urinary occult blood to nephrotic syndrome or acute kidney injury. LN is associated with considerable morbidity and mortality [1].

Cytokines play a key role in disease initiation and progression; in fact, in the kidney, immune complex deposition activates mesangial cells. Once activated by immune complexes and/or autoantibodies, renal resident cells secrete cytokines that may further amplify inflammatory processes [2].

\section{Case Report}

A 42-year-old woman presented with LN due to SLE. She was first admitted to the Nephrology and Dialysis Department of San Benedetto del Tronto Hospital in 2003 due to the detection of urinary abnormalities and increased creatinine (up to $3 \mathrm{mg} / \mathrm{dl}$ ). She had a presumptive diagnosis of psoriatic arthritis since 2002. An in-depth diagnostic and biopsy analysis led to the definitive diagnosis of SLE with LN [medical report of optical microscopy ascribable to LN (class II according to the WHO) with activities 7 and stage 0 , medical report of electronic microscopy compatible with the diagnosis of LN (class III according to the WHO) and antiphospholipid syndrome (APS) with presence of lupus anticoagulant antibodies and anticardiolipin antibodies].

Subsequently, the patient was subjected to treatment with induction immunosuppressive therapy with cyclophosphamide and prednisone for periodic exacerbation of basic immunological disease when she presented with proteinuria, high levels of inflammatory markers and abnormal liver function. She also had periods of clinical stability (characterized by a general improvement with normalization of liver and kidney function and remission of proteinuria) with mycophenolate mofetil.

These cycles continued until 2006, when she presented with hemolytic-uremic syndrome with severe hypertension $(260 / 130 \mathrm{~mm} \mathrm{Hg})$, grand mal and oliguria. She was then started on hemodialysis due to the rapid deterioration of renal function which, despite a new cycle of induction therapy, produced end-stage renal disease and required chronic hemodialysis treatment.

During the initial hemodialysis period, she continued therapy with mycophenolic acid and prednisone. Symptoms and signs of systemic disease activity persisted and included arthralgia, asthenia, episodic fever, maculopapular rash, elevated erythrocyte sedimentation rate and leuko-thrombocytopenia. Although the patient underwent 1-4 plasma exchanges (PEX) per month, the combination of PEX with methylprednisolone bolus and IgG admin- 


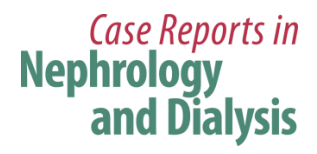

\begin{tabular}{l|l}
\hline Case Rep Nephrol Dial 2015;5:106-112 \\
\hline DOI: $10.1159 / 000381395$ & $\begin{array}{l}\text { C 2015 S. Karger AG, Basel } \\
\text { www.karger.com/cnd }\end{array}$ \\
\hline
\end{tabular}

Solano et al.: Radical Improvement of Signs and Symptoms in SLE when Treated with Hemodiafiltration with Endogenous Reinfusion Dialysis

istration achieved only limited improvements of arthralgia and cutaneous manifestations (necrotic-like skin lesions).

During the last 2 years, the patient has been started on a new hemodiafiltration technique, hemodiafiltration with endogenous reinfusion dialysis treatment, which uses the super-high-flux membrane Synclear 02 (SUPRA treatment) coupled to an adsorbent cartridge.

Fever and joint pain were significantly reduced as early as in the first week of treatment. During the following few months, skin manifestations were significantly reduced and the patient reported an improved quality of life (QoL). After starting SUPRA (3 times weekly for $4 \mathrm{~h}$ per session), the patient no longer needed any additional PEX treatment. Prednisone and immunosuppressors were gradually reduced and eventually discontinued since the improvement of symptoms suggested a trend towards systemic remission.

After 6 months of prednisone and mycophenolic acid therapy suspension, there were no further events to be considered as an expression of SLE activity. The patient has reported an improvement in asthenia and has not experienced any further episodes of fever or arthralgia. She currently continues using the SUPRA technique.

Laboratory analysis showed an improvement in leuko-thrombocytopenia; however, the patient still has a persistent elevation of the erythrocyte sedimentation rate, low levels of C3 and C4 complement and a moderate increase in IgG and antinuclear antibody.

The QoL of the patient was analyzed by the RAND 36-Item Health Survey before starting and 3 weeks after SUPRA treatment. The resulting scores illustrate how the SUPRA treatment led to an improvement in QoL. In particular, physical functioning, energy/fatigue and pain scores increased from 20 to 90,15 to 70 and 22.5 to 67.5 , respectively.

Although the timing between the beginning of SUPRA treatment and the complete remission of SLE symptoms may suggest an association between the SUPRA therapy and clinical improvement, we cannot exclude that there was a spontaneous remission of systemic disease, as known to occur sometimes in dialysis patients. This case, moreover, places further emphasis on the role of inflammatory mediators in SLE symptom manifestations and the therapeutic potential of SUPRA treatment.

\section{Discussion}

LN increases the morbidity and mortality of patients with SLE. SLE patients commonly have increased levels of IL- 6 and IL-10, which further increase in patients with active disease.

Jacob and Stohl [3] have rated four cytokines [IL-6, tumor necrosis factor $\alpha$ (TNF- $\alpha$ ), IFN- $\alpha$ and B-lymphocyte stimulator (BLyS)] as therapeutic targets in SLE. This study shows that the reduction of these proinflammatory cytokines has a beneficial effect on the course of the disease. To date, the potential of cytokines as therapeutic targets for lupus has been shown both by a BLyS inhibitor (belimumab, recently approved by the United States Food and Drug Administration as a treatment for SLE) and by ongoing trials on other cytokine inhibitors [4].

In particular, IL-6 is a multifunctional cytokine produced in response to inflammatory stimuli, including those mediated by IL-1 and TNF- $\alpha$, with key roles in the regulation of the immune response to infections. IL- 6 was indicated as a potent stimulator of the differentiation and activation of lymphoid and myeloid cells and the production of acute-phase proteins in the liver. IL- 6 is also a key regulator of many other cellular processes, including erythropoiesis, neuronal cell differentiation and bone metabolism. There is an inverse correlation between levels of IL-6 and hemoglobin levels in patients with SLE. 
Solano et al.: Radical Improvement of Signs and Symptoms in SLE when Treated with Hemodiafiltration with Endogenous Reinfusion Dialysis

Hemodiafiltration with on-line endogenous reinfusion (HFR-SUPRA treatment) is a dialytic method which combines the processes of diffusion, convection and adsorption. The performance of this system is linked to the optimal combination of the membrane permeability and cartridge resin bed [5].

It has been demonstrated that the sorbent cartridge has a high affinity for several uremic toxins and middle molecules, such as $\beta 2$-microglobulin, homocysteine, angiogenin, leptin, parathormone and some chemokines and cytokines. In contrast, the cartridge does not retain bicarbonate, amino acids and hydrosoluble vitamins. Other solutes, such as urea, creatinine, uric acid, $\mathrm{Na}+\mathrm{K}+, \mathrm{Ca} 2+$, phosphate and bicarbonate, which are not adsorbed by the cartridge, are cleared, by diffusion, in the second chamber of the filter [6-8].

In addition to cytokine removal information, high-performance liquid chromatography coupled with quadrupole time-of-flight mass spectrometer (HPLC-QTOF-MS) was used for the identification of proteins in the ultrafiltrate and the species captured by the resin bed (eluted proteins), obtained in a dialysis session of the patient (fig. 1; table 1).

Several biomarker of LN kidney injuries were identified in HPLC-QTOF analysis of eluted proteins, such as retinol-binding protein 4 (RBP4), neutrophil gelatinase-associated lipocalin (NGAL), zinc $\alpha-2$ glycoprotein (ZA2G) and cystatin-C (CYTC).

Moreover, additional uremic toxins such as serotransferrin (TRFE), $\alpha$-1-acid glycoprotein (A1AG1), prostaglandin-H2 D-isomerase (PTGDS) and transthyretin (TTHY) were identified. All of these markers are considered, by Varghese et al. [9], as biomarkers for glomerular disease. Furthermore, Suzuki et al. [10] initiated the validation of TRFE, ceruloplasmin (CERU), A1AG1, PTGDS, albumin and albumin-related fragments as biomarkers for active pediatric LN. Clearly, we identified several fragments of immunoglobulins that are implicated in the etiopathogenesis of SLE.

Another important protein in the pathophysiology of SLE is $\beta$-2-glycoprotein 1 (APOH), the principal autoantigen in APS. APS is associated with arterial and venous thrombosis, characterized by up to 30 different autoantibodies [11]. APOH, an abundant plasma phospholipid-binding protein, binds to anionic zwitterionic phospholipids, involved in clotting, but its physiological function is not completely understood [12].

Last but not least, we found neutrophil defensin 1 (DEF1) among the identified proteins, a member of the $\alpha$-defensin family. DEF1 - and DEF2 - has antibacterial, fungicide and antiviral activities. Both have antimicrobial activity against Gram-negative and Gram-positive bacteria. Defensins are thought to kill microbes by permeabilizing their plasma membrane [13].

In addition to exerting direct antimicrobial effects, defensins facilitate and amplify subsequent innate and adaptive immune responses, such as activation and degranulation of mast cells, interleukin and TNF production and maturation of dendritic cells.

Sthoeger et al. [14] have shown that the presence of high serum levels of $\alpha$-defensin correlates with higher rates of arthritis and renal and dermatological involvement and, in general, the $\alpha$-defensin levels correlate significantly with disease activity. Thus, when $\alpha$-defensin levels decreased to the normal range, an improvement in lupus-related manifestations was observed.

If compared with patients with low $\alpha$-defensin levels, most SLE patients with high levels of DEF1 were treated with corticosteroids. Probably, the elimination of this protein from blood could explain the reduction in corticosteroid administration [14].

The results of this study demonstrate that styrenic resin retained several proteins implicated in the LN pathogenesis. This means that SUPRA treatment is a dialysis method that reduces inflammatory status, uremic toxin level and could improve LN prognosis may- 
Solano et al.: Radical Improvement of Signs and Symptoms in SLE when Treated with Hemodiafiltration with Endogenous Reinfusion Dialysis

be through the counterbalance of the immune-modulatory response and the reduction of the APS.

\section{Disclosure Statement}

The authors declare that they have no conflicts of interest. Marialuisa Caiazzo is a fulltime employee of Bellco S.r.l.

\section{References}

1 Borchers AT, Leibushor N, Naguwa SM, Cheema GS, Shoenfeld Y, Gershwin ME: Lupus nephritis: a critical review. Autoimmun Rev 2012;12:174-194.

2 Iwata Y, Furuichi K, Kaneko S, Wada T: The role of cytokine in the lupus nephritis. J Biomed Biotechnol 2011;2011:594809.

3 Jacob N, Stohl W: Cytokine disturbances in systemic lupus erythematosus. Arthritis Res Ther 2011;13:228.

-4 Poole BD, Niewold TB, Tsokos GC, Via CS: Cytokines in systemic lupus erythematosus. J Biomed Biotechnol 2012;2012:427824.

-5 Wratten ML, Ghezzi PM: Hemodiafiltration with endogenous reinfusion. Contrib Nephrol 2007;158:94-102.

6 Tetta C, Ghezzi PM, De Nitti C, Fiorenzi A, Cianciavicchia D, Gervasio R: New options for on-line hemodiafiltration. Contrib Nephrol 2002;137:212-220.

7 Borrelli S, Minutolo R, De Nicola L, De Simone E, De Simone W, Zito B, Guastaferro P, Nigro F, Iulianiello G, Credendino O, Bassi A, Leone L, Capuano M, Auricchio MR, Conte G: Effect of hemodiafiltration with endogenous reinfusion on overt idiopathic chronic inflammation in maintenance hemodialysis patients: a multicenter longitudinal study. Hemodial Int 2014;18:758-766.

8 Riccio E, Cataldi M, Minco M, Argentino G, Russo R, Brancaccio S, Memoli A, Grumetto L, Postiglione L, Guida B, Memoli B: Evidence that p-cresol and IL- 6 are adsorbed by the HFR cartridge: towards a new strategy to decrease systemic inflammation in dialyzed patients? PLoS One 2014;9:e95811.

$\checkmark 9$ Varghese SA, Powell TB, Budisavljevic MN, Oates JC, Raymond JR, Almeida JS, Arthur JM: Urine biomarkers predict the cause of glomerular disease. J Am Soc Nephrol 2007;18:913-922.

10 Suzuki M, Wiers K, Brooks EB, Greis KD, Haines K, Klein-Gitelman MS, Olson J, Onel K, O’Neil KM, Silverman ED, Tucker L, Ying J, Devarajan P, Brunner HI: Initial validation of a novel protein biomarker panel for active pediatric lupus nephritis. Pediatr Res 2009;65:530-536.

11 Shoenfeld Y, Twig G, Katz U, Sherer Y: Autoantibody explosion in antiphospholipid syndrome. J Autoimmun 2008;30:74-83.

12 Miyakis S, Giannakopulos B, Krilis SA: Beta 2 glycoprotein I - function in health and disease. Thromb Res 2004;114:335-346.

13 Ericksen B, Wu Z, Lu W, Lehrer RI: Antibacterial activity and specificity of the six human $\alpha$-defensins. Antimicrob Agents Chemother 2005;49:269-275.

14 Sthoeger ZM, Bezalel S, Chapnik N, Asher I, Froy O: High $\alpha$-defensin levels in patients with systemic lupus erythematosus. Immunology 2009;127:116-122. 
Case Reports in

Nephrology

and Dialysis

\begin{tabular}{l|l}
\hline \multicolumn{2}{l|}{ Case Rep Nephrol Dial 2015;5:106-112 } \\
\hline DOI: $10.1159 / 000381395$ & $\begin{array}{l}\text { C } 2015 \text { S. Karger AG, Basel } \\
\text { www.karger.com/cnd }\end{array}$ \\
\hline
\end{tabular}

Solano et al.: Radical Improvement of Signs and Symptoms in SLE when Treated with Hemodiafiltration with Endogenous Reinfusion Dialysis

Table 1. Proteins identified in resin eluate and ultrafiltrate by HPLC-QTOF-MS analysis

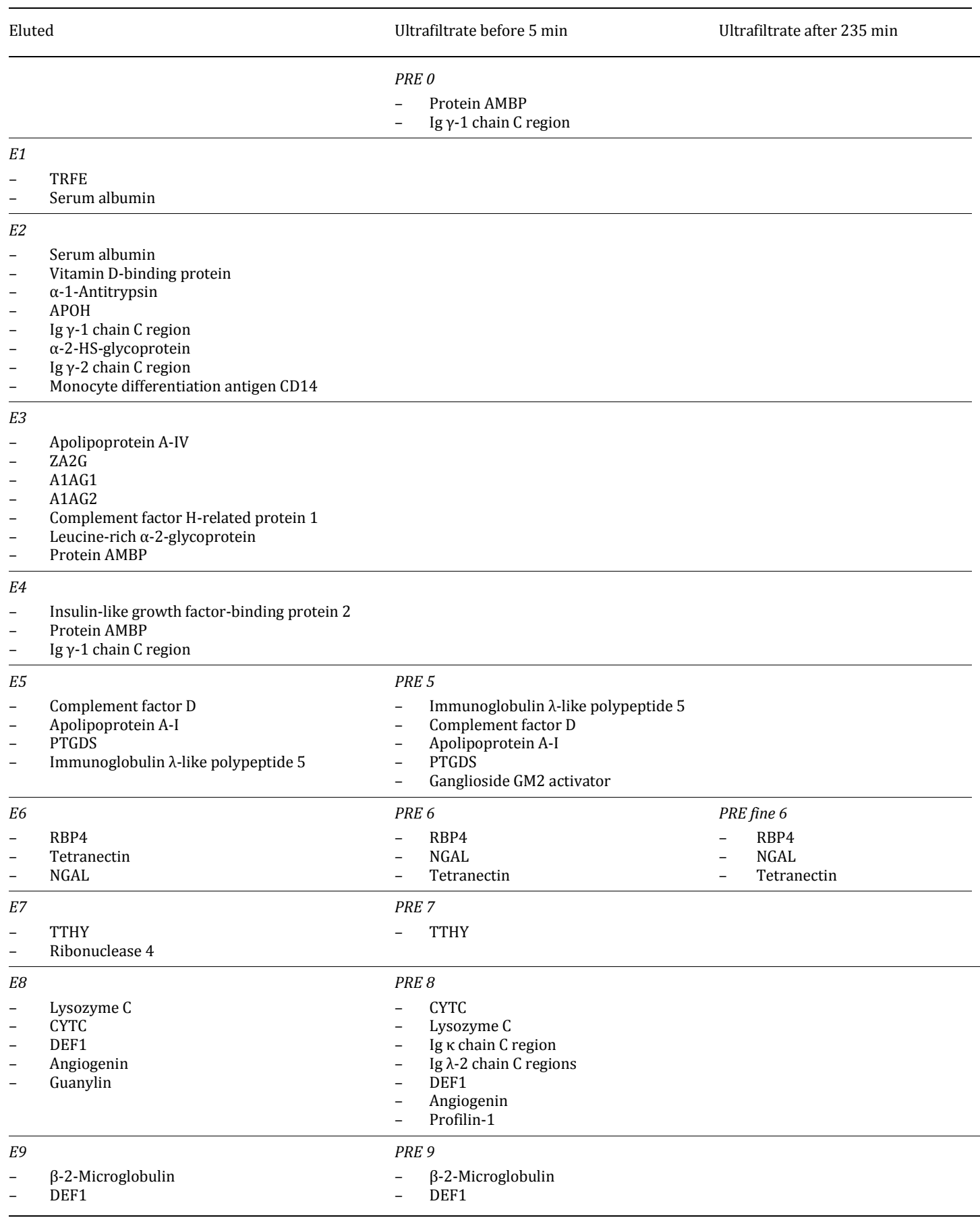


Case Reports in

Nephrology

and Dialysis

\begin{tabular}{l|l}
\hline \multicolumn{2}{l}{ Case Rep Nephrol Dial 2015;5:106-112 } \\
\hline DOI: 10.1159/000381395 & $\begin{array}{l}\text { ○ 2015 S. Karger AG, Basel } \\
\text { www.karger.com/cnd }\end{array}$ \\
\hline
\end{tabular}

Solano et al.: Radical Improvement of Signs and Symptoms in SLE when Treated with Hemodiafiltration with Endogenous Reinfusion Dialysis

kDa

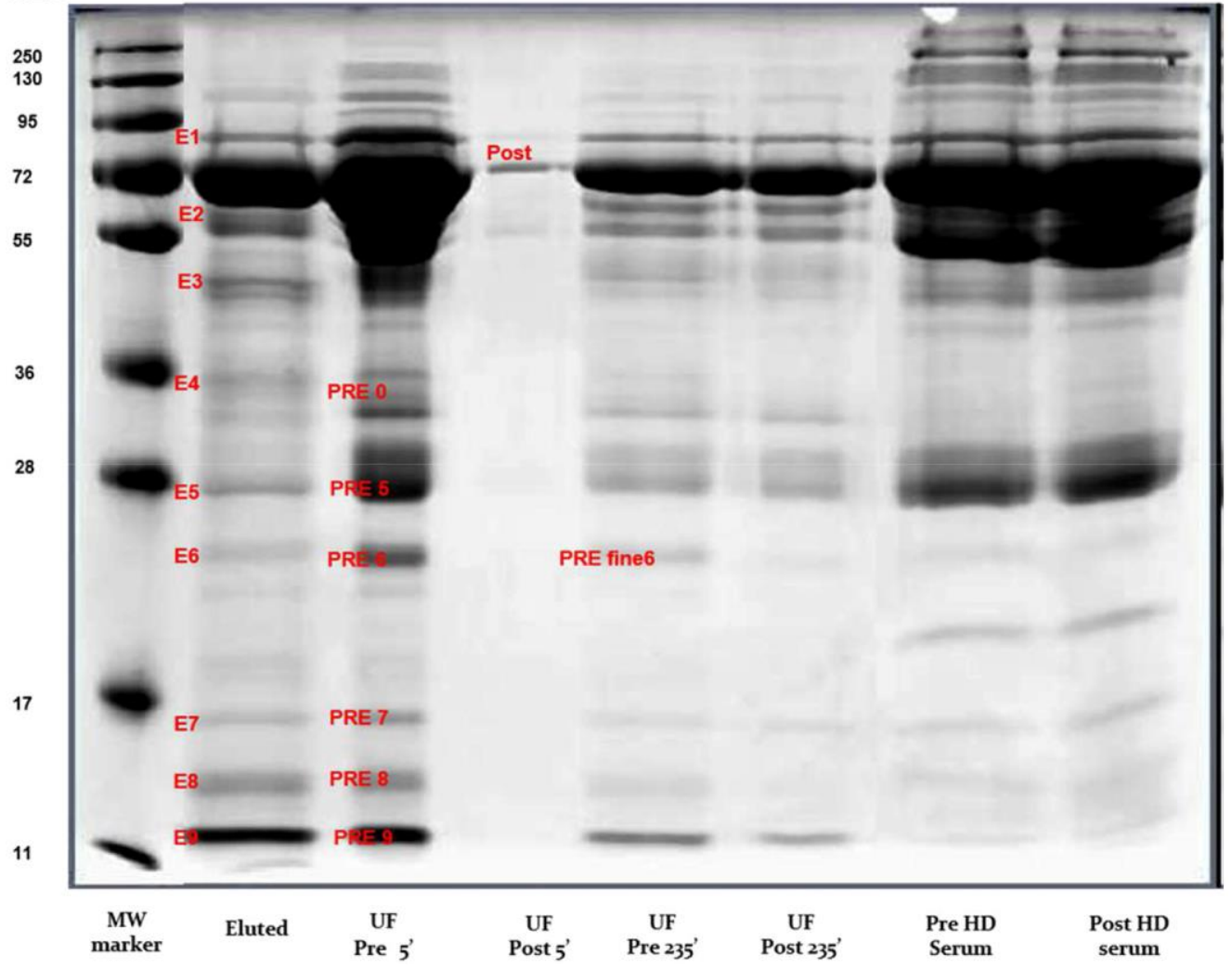

Fig. 1. SDS-PAGE of proteins captured by resin bed (eluted proteins), ultrafiltrate before and after cartridge at the beginning ( $5 \mathrm{~min}$ ) and at the end of treatment (235 $\mathrm{min}$ ) and serum before and after dialysis. 